\title{
Magnetic Interaction in FeCo Alloy Nanotube Array
}

\author{
D. Zhou ${ }^{1}$, T. Wang' ${ }^{2}$ M. G. Zhu ${ }^{1}$, Z. H. Guo ${ }^{1}$, W. $\mathrm{Li}^{1 *}$, and F. S. $\mathrm{Li}^{2}$ \\ ${ }^{1}$ Division of Functional Materials, Central Iron \& Steel Research Institute, Beijing 100081, China \\ ${ }^{2}$ Key Laboratory for Magnetism and Magnetic Materials of the Ministry of Education, Lanzhou University, Lanzhou 730000, China
}

(Received 2 August 2011, Received in final form 30 September 2011, Accepted 5 October 2011)

\begin{abstract}
An array of FeCo nanotubes has been successfully fabricated in the pores of porous anodic aluminum oxide (AAO) templates by wetting templates method. The morphology and structure of the nanotube array were characterized by scanning electron microscopy, transmission electron microscopy and $x$-ray diffraction. The average diameter of the nanotubes was about $200 \mathrm{~nm}$, and the length was more than $10 \mu \mathrm{m}$. Vibrating sample magnetometer and superconducting quantum interference device were used to investigate the magnetic properties of the nanotube array. Interaction between the nanotubes has been found to be demagnetizing as expected and the switching field distribution is broad.
\end{abstract}

Keywords : magnetic interaction, nanotube, FeCo alloy, switching field distribution

\section{Introduction}

The development of self-assembled nano materials, especially the application of AAO templates, has largely promoted the fabrication of novel nano structures. Researches on magnetic nanowires and their arrays prepared in pores of AAO templates have plenty of outcomes [15]. In recent years, with the development of the methods for preparing magnetic nanotubes, the synthesis of tubular nanostructures and the studies on the magnetic properties of nanotubes are pioneered in magnetic nanotechnology. The methods for fabrication include: electroless deposition [6], electrodeposition [7], wetting templates [8-12] and so on. Because of the potential applications demonstrated by previous studies, such as nanomedicine and biotechnology [13], ultrahigh-density magnetic storage devices [14], and nano electromechanical system device [15], magnetic nanotubes have attracted more and more attention.

Interactions always exist between the nano magnetic materials, which will affect the properties of the materials and their applications, just like the decrease of squareness ratio $(\mathrm{Mr} / \mathrm{Ms})$ in nanowire arrays [16], the increase of media noise in magnetic recording materials [17] and so on. So it is important to study the interactions between the

\footnotetext{
*Corresponding author: Tel: +86-10-62185125
}

Fax:+86-10-62185854, e-mail: magnet@cisri.cn magnetic units in the arrays. In this paper, the interaction effect taking place within the nanotube array is discussed.

\section{Experiment}

The FeCo nanotubes used in this letter were fabricated by wetting templates method and hydrogen reduction in porous alumina templates [8-12]. The advantages of this method in the production of magnetic and other inorganic nanotubes are obvious, because of its simplicity, convenience, and capability of producing large quantities of samples.

Transmission electron microscopy (TEM) and scanning electron microscopy (SEM) were used to confirm the morphology of the FeCo alloy nanotubes. The crystal structure of the nanotubes was examined by X-ray diffraction (Philips X'Pert, Holland). Vibrating sample magnetometer (VSM) and superconducting quantum interference device (SQUID) were used to measure the magnetic properties. The micromagnetic simulations were performed with the three-dimensional object oriented micromagnetic framework (3D OOMMF) program [18].

\section{Results and Discussion}

Fig. 1(a) and (b) are SEM image of FeCo nanotube array in the AAO template and TEM image of FeCo nanotubes released from alumina, respectively. The uni- 


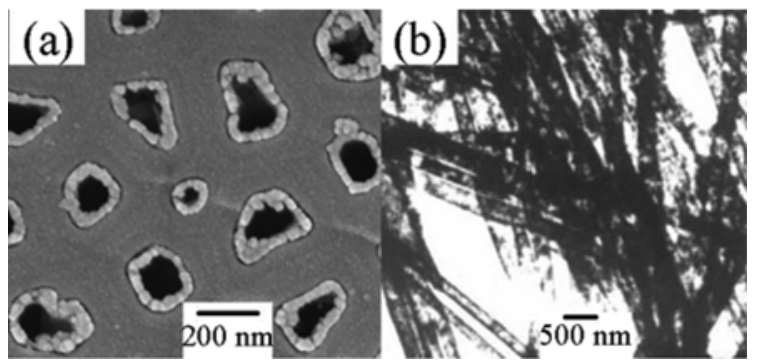

Fig. 1. (a) SEM image of an FeCo nanotube array, (b) TEM images of A bunch of FeCo nanotubes.

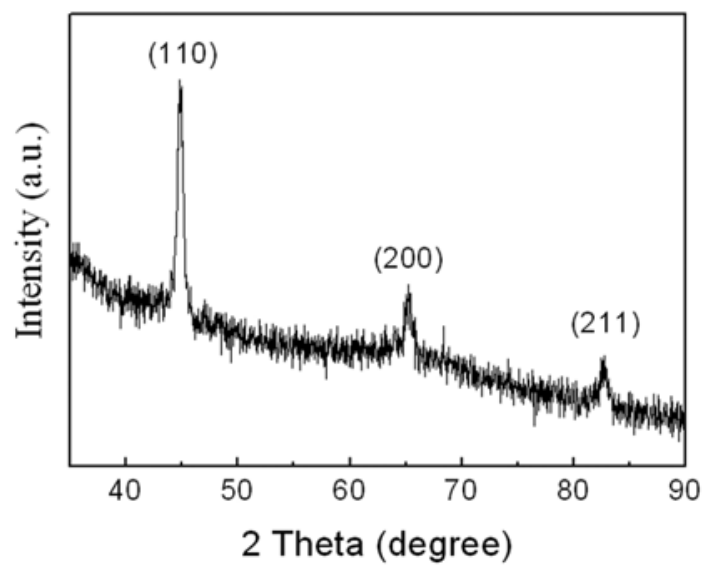

Fig. 2. X-ray diffraction pattern of CoFe nanotube array with AAO template.

formity of the nanotube array depends on the quality of AAO template, and a perfect hexagonal pore arrangement can be formed with the employment of imprint lithography technology [10]. Fig. 2 shows the x-ray diffraction pattern of the FeCo nanotube array, which reveals that the crystal structure of FeCo alloy is a body-centered cubic.

Fig. 3 shows hysteresis loops of FeCo alloy nanotube array, measured by superconducting quantum interference device (SQUID) magnetometry with the applied fields parallel and perpendicular to the tube axis at $10 \mathrm{~K}$ and $300 \mathrm{~K}$. As it can be seen, the coercivity at $10 \mathrm{~K}$ is larger than that measured at $300 \mathrm{~K}$. The coercivity change with temperature mainly depends on the saturation magnetization and magnetocrystalline anisotropy at low temperature. Because of the high aspect ratio of the nanotube, when the external field is applied, the demagnetization energy in the direction along the tube axis is much smaller than perpendicular to the tube axis, and the sample could be magnetized more easily along the tube axis (see fig. 3). Fig. 4 shows the micromagnetic simulation result of the moment distribution in one $\mathrm{FeCo}$ nanotube, which is consistent with Mössbauer spectrum

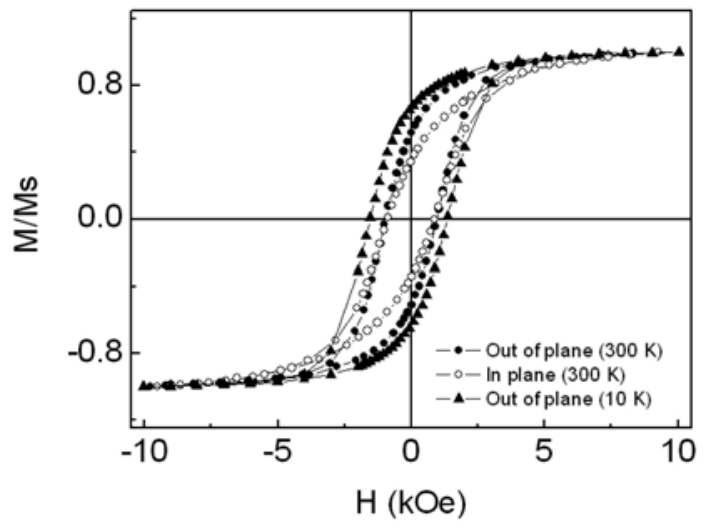

Fig. 3. Magnetic hysteresis curves of the FeCo nanotube array at $10 \mathrm{~K}$ and $300 \mathrm{~K}$. (Out of plane means the applied field is parallel to tube axis; in plane means the field is perpendicular to the tube axis.)

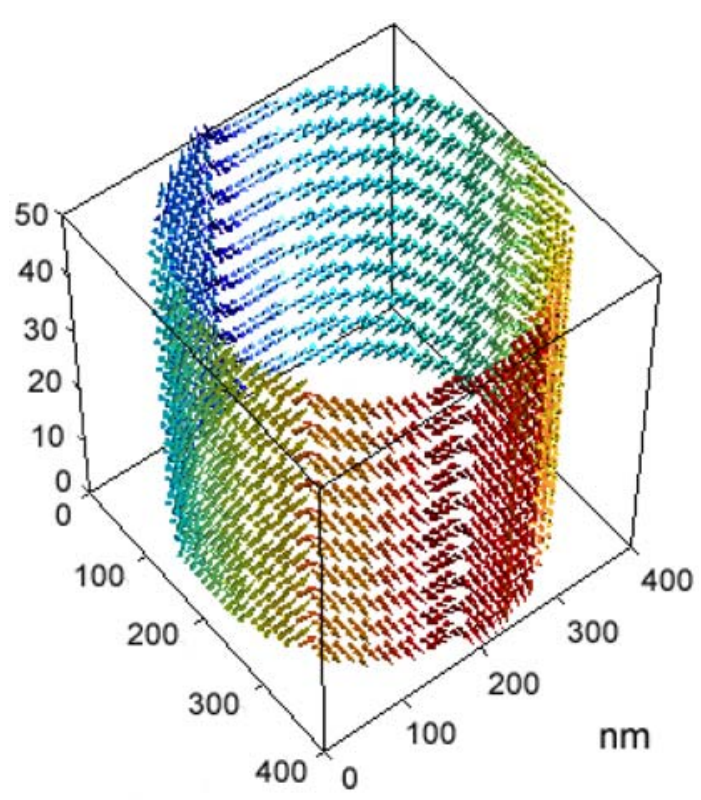

Fig. 4. (Color online) Micromagnetic simulation results for an infinitely long FeCo nanotube.

data [12]: the magnetic moments in the nanotubes are aligned mainly in the plane of tube walls, and the distributions of the magnetic moment in the plane are isotropic.

Fig. 5 shows the isothermal remanent magnetization (IRM) and DC de-magnetization (DCD) with applied field parallel to the tube axis at room temperature. The study of remanence curves (IRM and DCD) is a commonly used method to determine the energy barrier distribution in nano materials systems [19]. It is important to note that, in general, $\mathrm{M}(\mathrm{H})$ loops are determined by an admixture of both reversible and irreversible magnetization processes. In contrast, remanent magnetization loops 


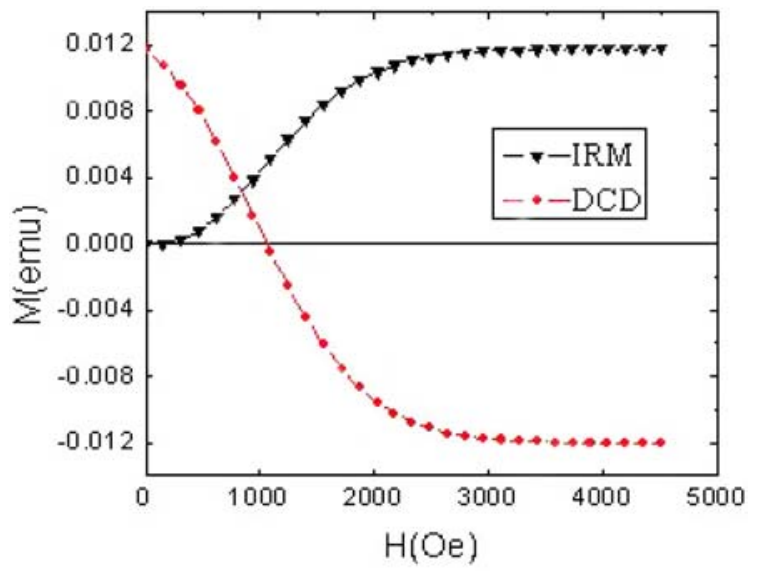

Fig. 5. (Color online) Isothermal remanent magnetization (IRM) and DC de-magnetization (DCD) curves for FeCo nanotube array with applied field parallel to the tube axis.

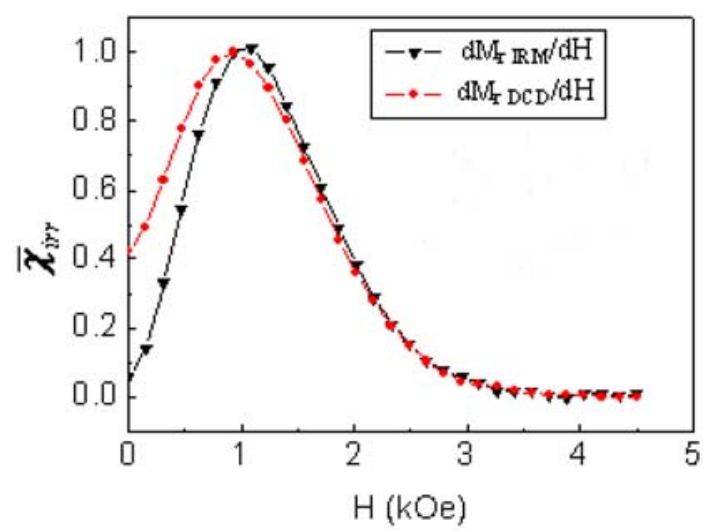

Fig. 6. (Color online) Normalised $\chi_{\text {irr }}$ obtained from the derivative of IRM curve and DCD curve with applied field parallel to tube axis.

just demonstrate irreversible processes. Fig. 6 illustrates the irreversible susceptibility curves, $\chi_{\text {irr }}$, which are the derivatives of the DCD and IRM curves with respect to applied field. From irreversible susceptibility curves, we can get the switching fields distribution (SFD). In general terms a broad SFD is indicative of wide variation in the micromagnetic environment within the magnetic material [20]. It can be seen from Fig. 6 that the SFD is broad and this may be caused by the defects in nanotubes and the diameter distribution of the nanotubes. $\chi_{\text {irr }}$ curve obtained from the DCD curve is finite at zero field, indicating that at room temperature a considerable proportion of the particles are superparamagnetic and do not contribute to the remanence [19]. As can be seen in Fig. 1, the nanotubes are composed of nanograins, some of which could be smaller than the critical size of superparamagnetism. The exchange coupling may not be strong enough to suppress superparamagnetism. The paramagnetic

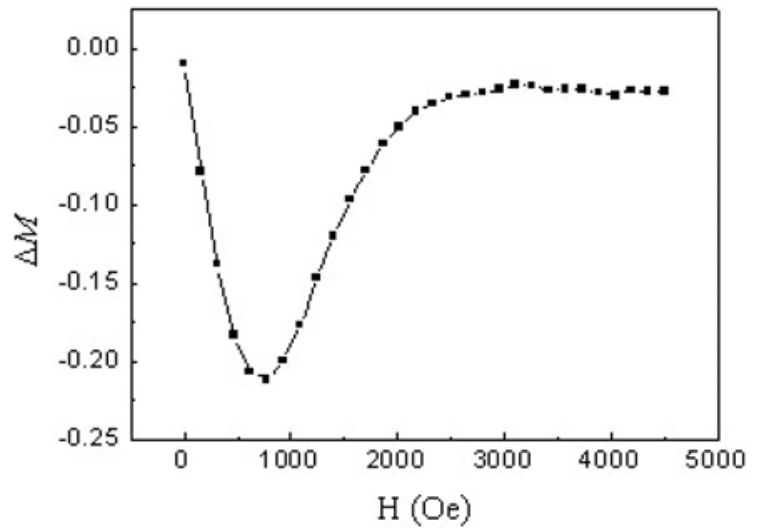

Fig. 7. Normalized $\Delta M$ plot for the FeCo nanotube array with the field applied parallel to the tube axie at room temperature.

quadrupole doublet in Mössbauer spectrum also showed the appearance of superparamagnetic particles [12, 21].

Henkel Plot and $\Delta M$ plot are widely used to study the interactions between the nanoscale materials $[17,20,22$, 23]. Here, $\Delta M$ plot is employed to study the magnetic interaction in FeCo alloy nanotube array. The equation of $\Delta M$ plot can be written as: [17]

$$
\Delta M(H)=\bar{M}_{d}(H)-\left[1-2 \bar{M}_{r}(H)\right]
$$

Fig. 7 shows the $\Delta M$ plot curve with the applied field parallel to the tube axis. A positive value of $\Delta M$ corresponds to interactions promoting the magnetized state, whereas a negative value would be due to demagnetizing interactions. The figure shows a negative value for the quantity $\Delta M$. This indicates that the interactions taking place between the nanotubes are demagnetizing, such as dipolar interactions. The demagnetization of the nanotube array is easier than magnetization.

\section{Conclusions}

A high quality FeCo array was successfully prepared in pores of anodic aluminum oxide template. When the magnetization direction is parallel to the tube axis, demagnetizing interactions occur in the array, because of the flux closure configurations between nanotubes. Due to both the defects in nanotubes and the diameter distribution of the nanotube, the irreversible moment switching field distribution (SFD) is wide.

\section{Acknowledgements}

We gratefully acknowledge the support of the National Basic Research Program of China (2010CB934601), National Natural Science Foundation of China (50931001, 
11004038) and the National High Technology Development Program of China (2010AA03A401).

\section{References}

[1] X. Y. Zhang, L. H. Xu, J. Y. Dai, and H. L. W. Chan, Physica B 353, 187 (2004).

[2] J.-R. Choi, S. J. Oh, H. Ju, and J. Cheon, Nano Letters 5, 2179 (2005).

[3] F. S. Li, T. Wang, L. Y. Ren, and J. R. Sun, J. Phys.: Condens. Matter 16, 8053 (2004).

[4] P. M. Paulus, F. Luis, M. Kröll, G. Schmid, and L. J. de Jongh, J. Magn. Magn. Mater. 224, 180 (2001).

[5] S. G. Yang, H. Zhu, D. L. Yu, Z. Q. Jin, S. L. Tang, and Y. W. Du, J. Magn. Magn. Mater. 222, 97 (2000).

[6] S. C. Lin, S. Y. Chen, S. Y. Cheng, and J. C. Lin, Solid State Sci. 7, 896 (2005).

[7] J. C. Bao, C. Y. Tie, Z. Xu, Q. F. Zhou, D. Shen, and Q. Ma, Adv. Mater. 13, 1631 (2001).

[8] Y. C. Sui, R. Skomski, K. D. Sorge, and D. J. Sellmyer, Appl. Phys. Lett. 84, 1525 (2004).

[9] P. Aranda and J. M. García, J. Magn. Magn. Mater. 249, 214 (2002).

[10] K. Nielsch, F. J. Castaño, S. Matthias, W. Lee, and C. A. Ross, Adv. Eng. Mater. 7, 217 (2005).
[11] F. S. Li, D. Zhou, T. Wang, Y. Wang, L. J. Song, and C. T. Xu, J. Appl. Phys. 101, 014309 (2007).

[12] D. Zhou, Z. W. Li, X, Yang, F. S. Wen, and F. S. Li, Chin. Phys. Lett. 25, 1865 (2008).

[13] R. Gasparac, P. Kohli, M. O. Mota, L. Trofin, and C. R. Martin, Nano Letters 4, 513 (2004).

[14] S. Khizroev, M. H. Kryder, D. Litvinov, and D. A. Thompson, Appl. Phys. Lett. 81, 2256 (2002).

[15] M. Zahn, J. Nanopart. Res. 3, 73 (2001).

[16] H. Riccardo, J. Appl. Phys. 90, 5752 (2001).

[17] P. E. Kelly, K. O. Grady, P. I. Mayo, and R. W. Chantrell, IEEE Trans. Magn. 25, 3881 (1989).

[18] M. J. Donahue and D. G. Porter, OOMMF User's Guide Version 1.2a3 (2002) (http://math.nist.gov/oommf).

[19] M. Blanco-Mantecón and K. O. Grady, J. Magn. Magn. Mater. 296, 124 (2006).

[20] G. P. Heydon, S. R. Hoon, A. N. Farley, S. L. Tomlinson, M. S. Valera, K. Attenborough, and W. Schwarzacher, J. Phys. D: Appl. Phys. 30, 1083 (1997).

[21] G. F. Goya, T. S. Berquóand, and F. C. Fonseca, J. Appl. Phys. 94, 3520 (2003).

[22] A. Robinson and W. Schwarzacher, J. Appl. Phys. 93, 7250 (2003).

[23] E. P. Wohlfarth, J. Appl. Phys. 29, 595 (1958). 